\title{
Efficient polarization beam splitter pixels based on a dielectric metasurface: supplementary material
}

\author{
M. Khorasaninejad, ${ }^{1 *}$ W. Zhu, ${ }^{1}$ K. B. Crozier, $, 1,2,3 *$ \\ ${ }^{1}$ School of Engineering and Applied Sciences, Harvard University, 33 Oxford Street, Cambridge, MA 02138, USA \\ ${ }^{2}$ Department of Electrical and Electronic Engineering, University of Melbourne, Victoria 3010, Australia \\ ${ }^{3}$ School of Physics, University of Melbourne, Victoria 3010, Australia \\ *Corresponding authors: khorasani@seas.harvard.edu and kcrozier@unimelb.edu.au \\ Published 15 April 2015
}

This document provides supplementary information to "Efficient polarization beam splitter pixels based on a dielectric metasurface," http://dx.doi.org/10.1364/optica.2.000376. (C) 2015 Optical Society of America http://dx.doi.org/10.1364/optica.2.000376.s001

\section{Scanning Electron Micrographs of Single Nanoridges}

Scanning electron micrographs (SEMs) of successively rotated nanoridges are shown in Fig. S1. All nanoridges have widths $W=60$ $\mathrm{nm}$, lengths $L=5 \mu \mathrm{m}$ and heights $H=500 \mathrm{~nm}$. Nanoridges successively rotate by 45 degree and are spaced by $5 \mu \mathrm{m}$ (centerto-center).

\section{Measurement Set-up}

Figure S2 shows the measurement set-up. The set-up is a homebuilt optical microscope with an infrared-LED (IR-LED) as the light source. The output of the LED is collected by a condenser and passes through a linear polarizer, and illuminates the pixelintegrated polarization splitter (PIXIP) sample. Light transmitted through the sample is collected by a microscope objective lens. The latter, in combination with a tube lens (focal length $f=15 \mathrm{~mm}$ ), forms an image on an infrared camera.

\section{Asymmetric Deflection}

One-sided deflection is realized using a unit cell comprising eleven nanoridges whose widths $W$ linearly increase from $40 \mathrm{~nm}$ to 90 $\mathrm{nm}$, from left to right (Fig. S3). The different phase delays that the nanoridges generate (owing to their different widths) result in the output comprising a tilted wavefront. The nanoridges have centerto-center distance $P=500 \mathrm{~nm}$ and heights $H=2 \mu \mathrm{m}$, and simulations are performed at $\lambda=950 \mathrm{~nm}$. While input light polarized parallel to the nanoridges is defected to the right side (Fig. S3, top), light polarized perpendicular to the ridges mainly remains undeflected (Fig. S3, bottom).

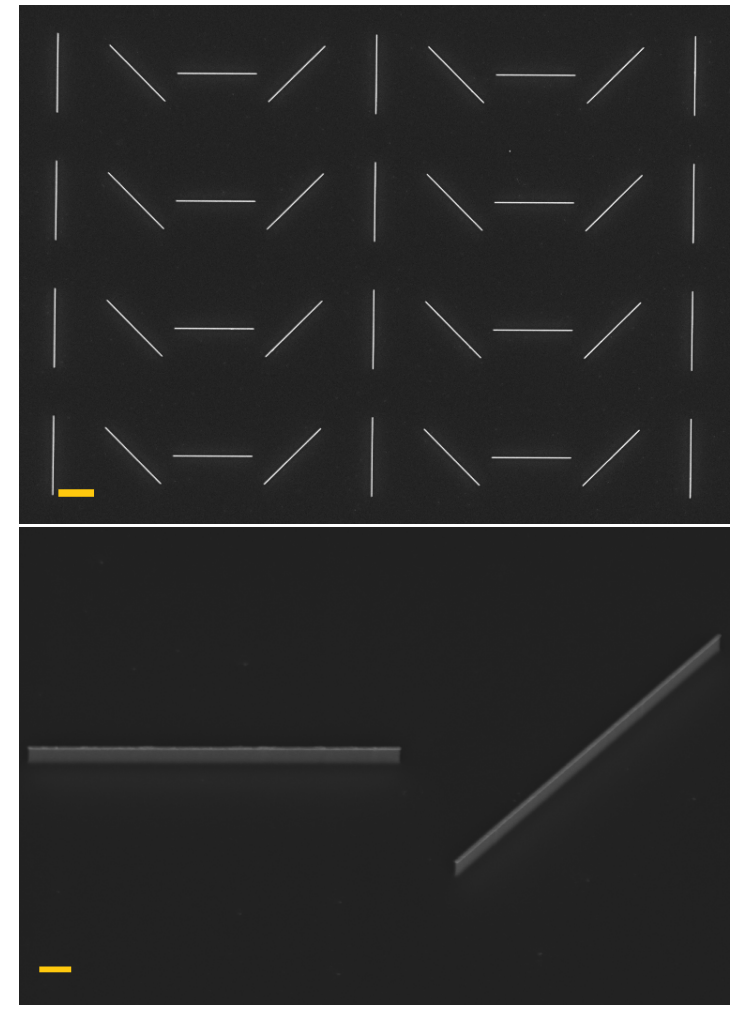

Fig. S1. Top: Top-view SEM of successively rotated single nanoridges, scale bar is $2 \mu \mathrm{m}$. Bottom: Tilted-view SEM of nanoridges, scale bar is $400 \mathrm{~nm}$. 


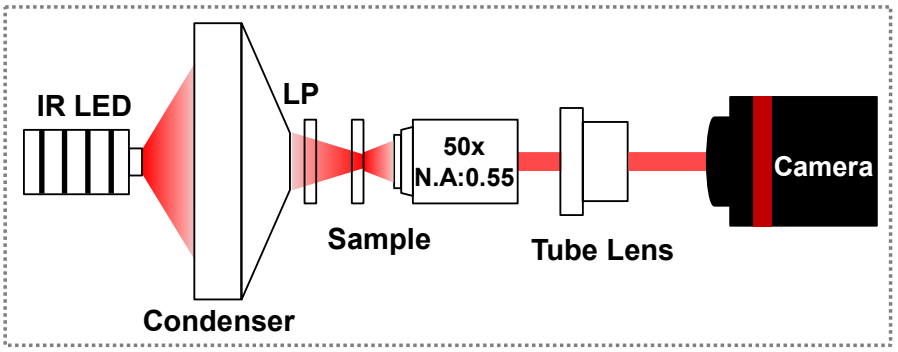

Fig. S2. Experimental set-up consisting of an LED $(\lambda=940 \mathrm{~nm})$ whose output is collected by a condenser and passes through a linear polarizer (LP). An objective lens (N.A:0.55, magnification: $50 \times$ ), a tube lens, and a camera are used to capture images.
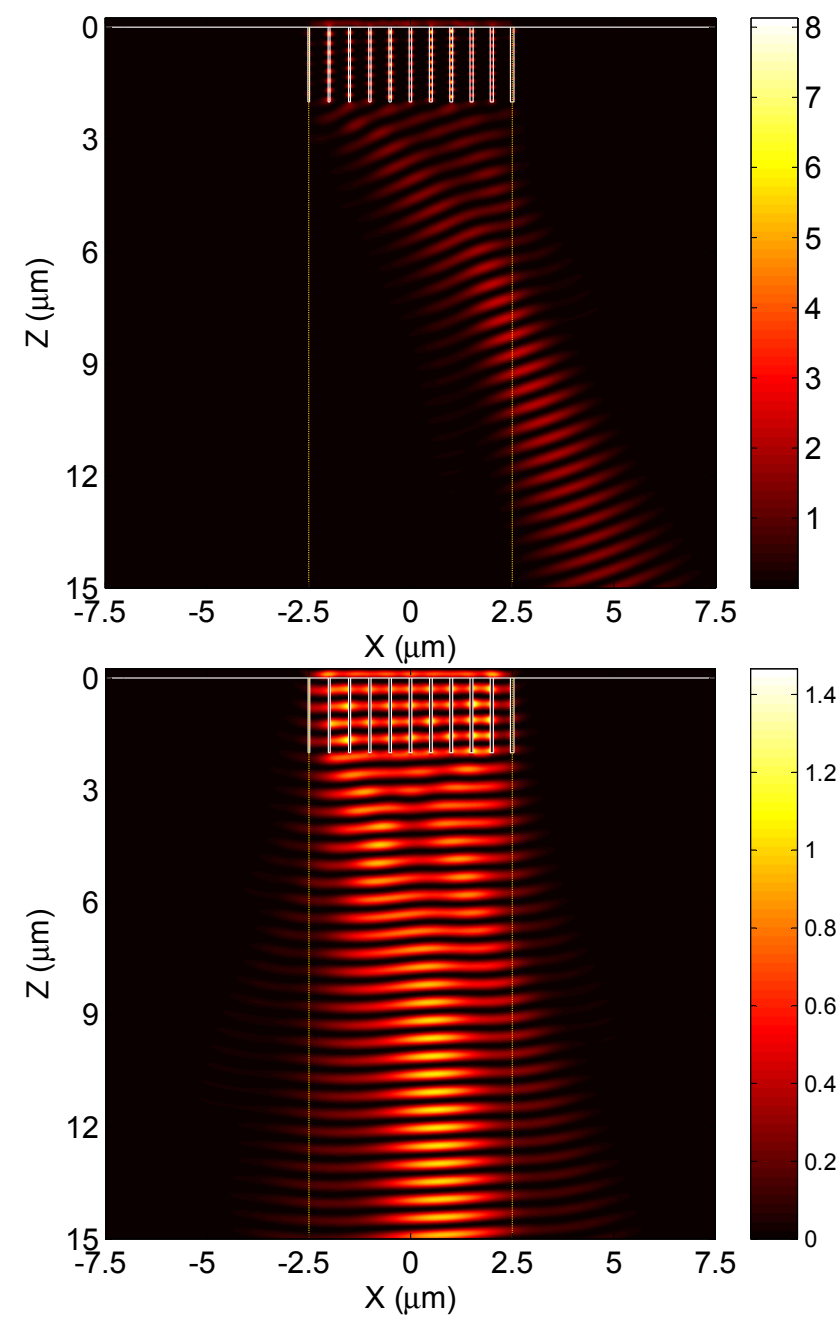

Fig. S3. Simulated power flow for light polarized parallel (top) and perpendicular (bottom) to length of nanoridges. Wavelength of operation is $\lambda=950 \mathrm{~nm}$. There are eleven nanoridges with heights $H=2 \mu \mathrm{m}$ and center-to-center distances $P=500 \mathrm{~nm}$. Width of far-left nanoridge is $W=40 \mathrm{~nm}$ and the width increases in a linear fashion $(\Delta W=5 \mathrm{~nm})$ to $W=90 \mathrm{~nm}$ for far-right nanoridge.

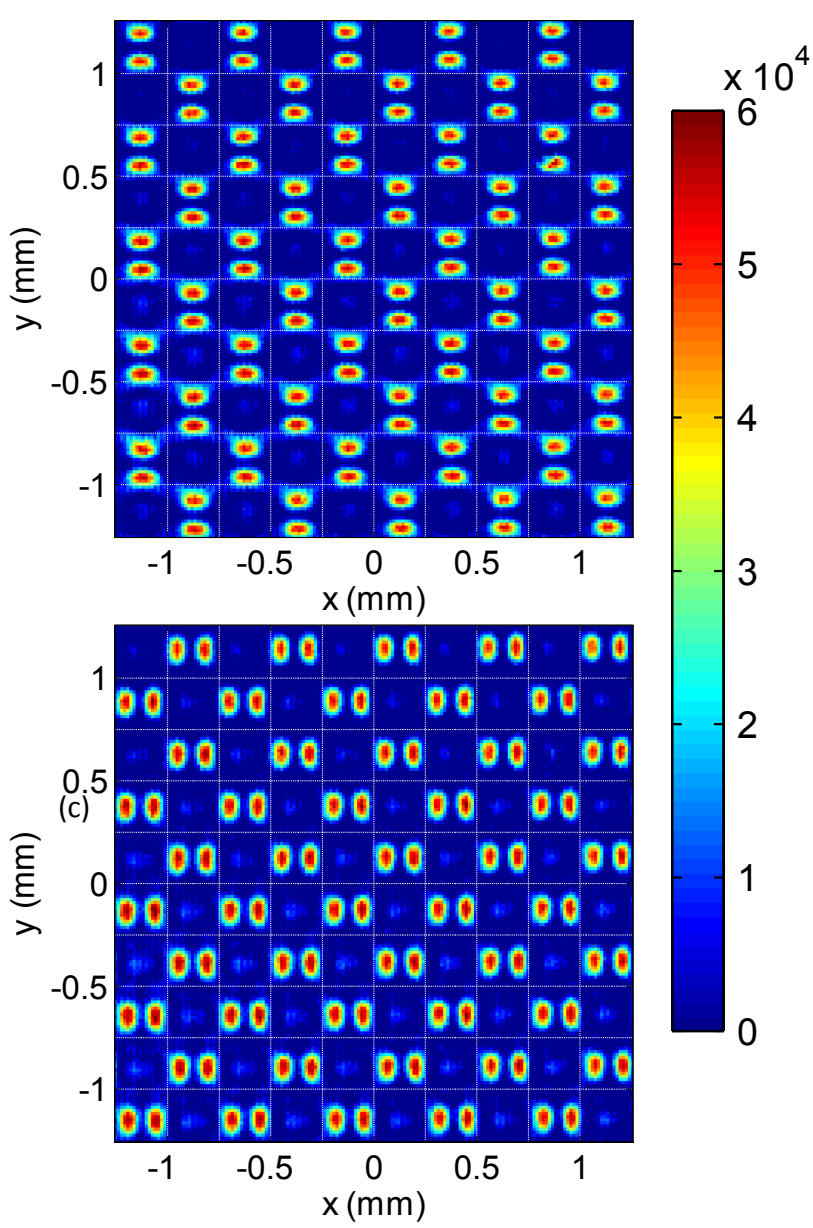

Fig. S4. Measured intensity distributions of PIXIP device (imaging plane is $15 \mu \mathrm{m}$ from substrate) showing PIXIP device steers impinging light. Top: input light is $x$-polarized. Bottom: input light is $y$-polarized.

\section{Intensity Distributions from PIXIP Device}

In Fig. S4, measured intensity distributions of region of PIXIP device containing $10 \times 10$ pixels $(5 \times 5$ unit cells $)$ are shown for input light having $x$ - (top figure) and $y$-polarizations (bottom figure), respectively.

\section{Evolution of Intensity Distribution as Input Polarization Changes}

In Fig. S5, the measured intensity distribution for one unit cells of the PIXIP device is shown for different choices of input polarization angle $\left(0\right.$ to $\left.90^{\circ}\right)$. Figure 5 of the main manuscript was generated by analysis of this data. 

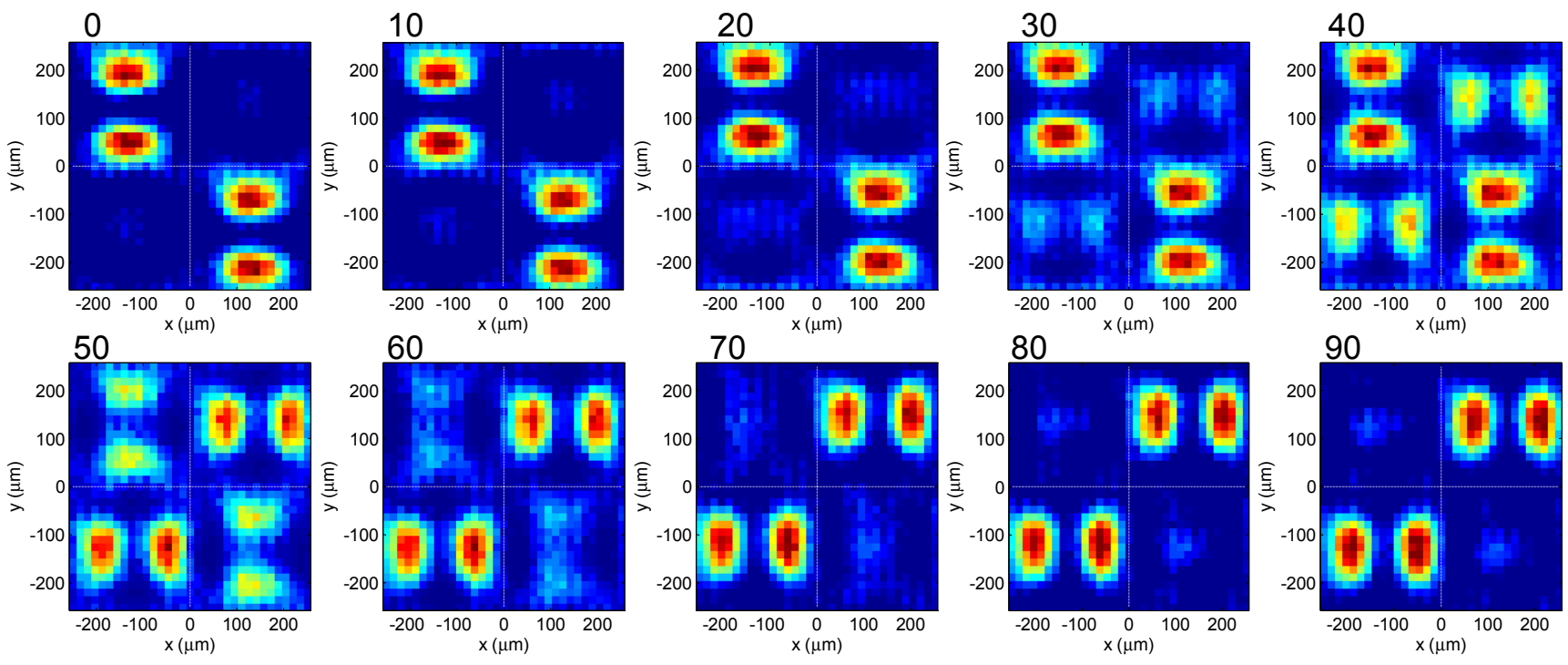

Fig. S5. Evolution of intensity distribution of one unit cell of PIXIP device when polarization is gradually changed from 0 to $90^{\circ}$. Polarization angle (in degrees) is noted at top of each image. 